\title{
Normalized-constrained approach for joint clearance design of deployable overconstrained Myard 5R mechanism
}

\author{
Hao SHEN*, Hailin HUANG** and Tie JI* \\ ${ }^{*}$ Room 522, Design Building, School of Design, Hunan University, Changsha, hunan, P.R. China, 410082 \\ Corresponding author: Dr. Hao Shen \\ E-mail: hao.shen@mlab.cn \\ ** Shenzhen Graduate School, Harbin Institute of Technology, Shenzhen, P. R. China, 518055
}

Received 15 July 2015

\begin{abstract}
This paper proposes a normalized-constrained approach for the joint clearance design of deployable overconstrained Myard 5R mechanism. Firstly, the overconstrained mobility characteristic of Myard 5R mechanism is analyzed, we show that the dimensional requirements are critical for this mechanisms such that the joint clearance is necessary and inevitable in the design process. Secondary, the normalized-constrained model of Myard mechanism without changing its deployable ability is proposed by attaching two revolute joints along the two short links of the original Myard mechanism, we show that this normalized-constrained mechanism is still deployable/foldable to the appropriate deployed/folded configurations under some possible dimensional errors. Thirdly, the clearance model for revolute joint is created, then we can calculate the optimized joint clearance based on the motion range of the two additional revolute joints in the normalized-constrained model. Finally, a design example is given to show the detailed design procedures of methodology proposed in this paper.
\end{abstract}

Key words : Overconstrained deployable mechanisms, Joint clearance, Normalized-constrained model

\section{Introduction}

Deployable mechanisms are widely used as the supporting structure of large spacecrafts(L. Puiga, A. et al. 2010). Due to the large volume characteristic of the space deployable mechanisms, multi-loop structures are always adopted in the design of this type of mechanisms because this type of structures are promise to have higher stiffness by comparing to their single-loop or open-loop counterparts. The multi-loop mechanisms are always kinematically overconstrained such that it is sensitive to dimensional errors, even a small error will make the mechanism could not be functioned normally (Hailin Huang, et al. 2013). In order to ensure the mobility of the mechanisms, joint clearance is necessary and inevitable in mechanism design. Joint clearance is also designed for the cost reduction of the manufacturing, prestressing force reduction and easy of assembly of the complicated overconstrained mechanisms (Jian Meng, et al., 2009). For deployable mechanisms work in the space environment, the joint clearance is necessary to make the mechanisms adapt to the possible thermal deformations which will change the dimensions of the mechanical parts of the deployable mechanisms. However, the joint clearance can also introduce some negative effect on the mechanisms, such as the reducing of the accuracy, introducing contact collision force and changing the mobility stability of the mechanisms etc. The Hubble telescope in USA and DFH-3 satellite in China have ever suffered from the negative dynamic effect due to the joint clearance (Nurre G S, et al., 1995, Foster C L, et al., 1995). Therefore, it is necessary to take the joint clearance into consideration when designing the large deployable mechanisms, proper and optimized joint clearance is very important for this type of mechanisms. This paper aims at dealing with the joint clearance design problem of overconstrained deployable mechanisms. The overconstrained mobility characteristic of Myard 5R mechanism, which we will use as the example in this paper, is first analyzed. The normalized-constrained model of Myard mechanism without changing its deployable ability is proposed by attaching two revolute joints along the two short links of the original Myard mechanism. After that, the clearance model for revolute joint is created, then we can calculate the optimized joint clearance based on the motion range of the two additional revolute joints in the 
normalized constrained model. Finally, a design example is given to show the design procedures of the methodology proposed in this paper.

The rests of this paper are organized as follows: in section 2, the overconstrained mobility characteristic of Myard $5 \mathrm{R}$ mechanism will first be analyzed, in section 3, the normalized-constrained model of Myard 5R mechanism is given and it's deployable ability under some possible dimensional errors is analyzed, the joint clearance model for revolute joint is created and the approach for calculating optimized clearance for Myard deployable mechanism is developed in section 4, a design example is given in section 5 and a conclusion is drawn in the last section.

\section{Mobility analysis of the Myard deployable mechanism}

The basic Myard mechanism is shown in Fig. 1, it consists of five revolute joints A, B, C, D and E, joint axes of A and $\mathrm{B}$ always intersect at a point $\mathrm{O}$, joint axes of $\mathrm{C}$ and $\mathrm{D}$ always intersect at another point $\mathrm{P}$, the mechanism is plane-symmetric around a plane determined by points $\mathrm{P}, \mathrm{O}$ and axis $\mathbf{s}_{5}$ of joint $\mathrm{E}$ (Myard F E., 1931). The Myard 5R mechanism is a typical overconstrained mechanism because its degree of freedom (DOF) could not be calculated by general Grübler-Kutzbach mobility criterion (Grigore Gogu, 2005). Based on reference Li B., et al. (2015), the two revolute joint axes $\mathbf{s}_{1}$ and $\mathbf{s}_{2}$ form a coplanar 2-twist screw system, then any rotation in the plane $\left\{\mathbf{s}_{1}, \mathbf{s}_{2}\right\}$ and passing through the point $\mathrm{O}$ can be realized for the two links $c$ and $d$. Similarly, any rotation in the plane $\left\{\mathbf{s}_{3}, \mathbf{s}_{4}\right\}$ and passing through the point $\mathrm{P}$ can be realized for the two links $c$ and $d$. For the closed loop Myard 5R mechanism, the two open loop kinematic chains in the two sides of the two links $c$ and $d$ should have non-empty rotational intersection so that the mechanism has continuous mobility. Because $\mathbf{s}_{5} \cap\left\{\mathbf{s}_{1}, \mathbf{s}_{2}\right\}=\mathrm{Q}$, then $\mathbf{s}_{5} \cap \mathbf{O Q}=\mathrm{Q}$ such that any rotation in the plane $\left\{\mathbf{s}_{5}, \mathbf{O Q}\right\}$ and passing through the point $\mathrm{Q}$ can also be realized. In other words, for the open loop kinematic chain consists of revolute joints $\mathrm{A}, \mathrm{B}$ and $\mathrm{E}$, the two links $c$ and $d$ can be rotated around axis that in the plane $\left\{\mathbf{s}_{5}, \mathbf{O Q}\right\}$ and passing through the point $\mathrm{Q}$. On the other hand, for the kinematic chain consists of revolute joint $\mathrm{C}$ and $\mathrm{D}$, the two links $c$ and $d$ can be rotated around axis that in the plane $\left\{\mathbf{s}_{3}, \mathbf{s}_{4}\right\}$ and passing through the point $\mathrm{P}$. Because the Myard 5R mechanism is symmetric around the plane determined by points $\mathrm{P}, \mathrm{O}$ and axis $\mathbf{s}_{5}$, then the plane $\left\{\mathbf{s}_{5}, \mathbf{O Q}\right\}$ passing through the point $\mathrm{P}$, and the point $\mathrm{Q}$ locates at the plane $\left\{\mathbf{s}_{3}, \mathbf{s}_{4}\right\}$, then the two links $c$ and $d$ in the closed loop mechanism have one allowable mobility around axis $\mathbf{P Q}$.

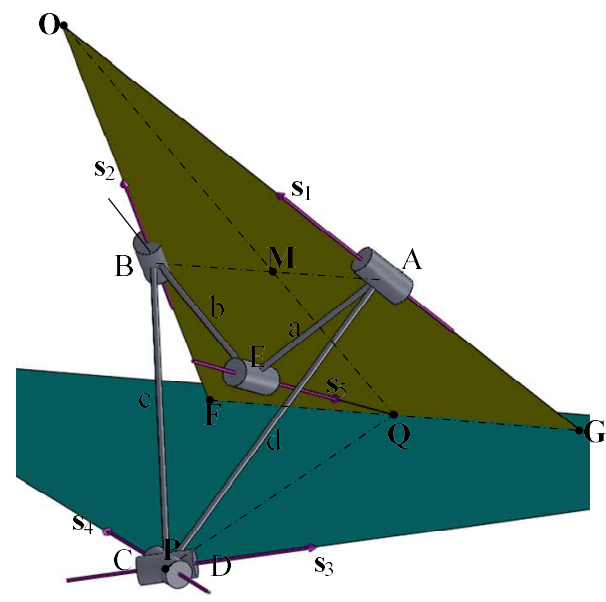

Fig. 1 Theoretical model of Myard mechanism

The Myard mechanism satisfies the following geometric constraints

$$
\left\{\begin{array}{l}
a=b, c=d \\
\alpha_{23}=\alpha_{41}=\pi / 2, \alpha_{51}=\pi-\alpha_{52}, \alpha_{34}=\pi-2 \alpha_{52} \\
R_{i}=0,(i=1,2,3,4,5) \\
a=d \sin \alpha_{52}
\end{array}\right.
$$

Where $\alpha_{i j}$ is angle of joint axes $\mathbf{s}_{i}$ and $\mathbf{s}_{j}$ around their common perpendicular, and $R_{i}$ is the offset of the adjacent two D-H links along axis $\mathbf{s}_{i}$.

Reference by Hailin Huang, et al., (2013) has shown that the Myard mechanism can be used as the basic unit for constructing large deployable mechanisms, the CAD model for a basic Myard deployable unit is as shown in Fig. 2(a). 
We have also made the physical prototype for this deployable mechanism, the deployed configuration is as shown in Fig. 2(b) and folded configuration is as shown in Fig. 2(c).

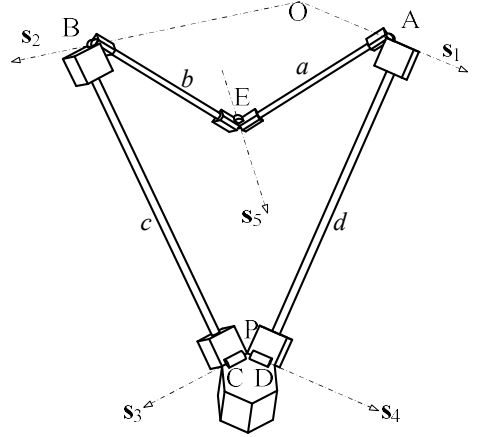

(a) CAD model

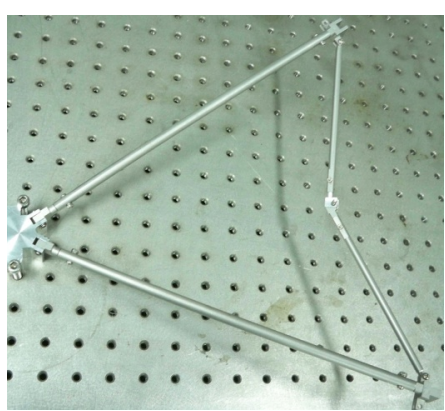

(b) Developed configuration

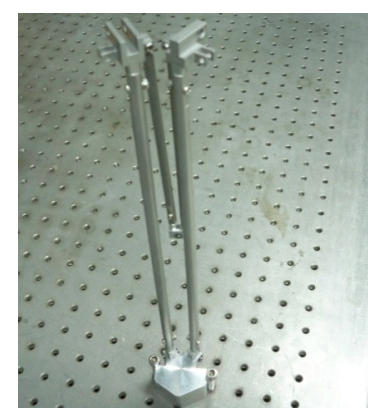

(c) Folded configuration

Fig. 2 Deployable Myard 5R mechanism

By using the Myard 5R mechanism as basic deployable units, we can construct the large deployable network as shown in Fig. 3, the deployable network can be infinitely extended and has single mobility, it can also be deployed from a bundle folded configuration (Fig. 3(a)) onto a planar deployed configuration (Fig. 3(c)).

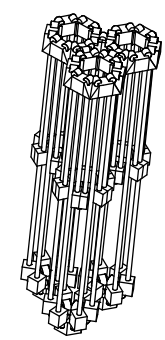

(a) Folded configuration

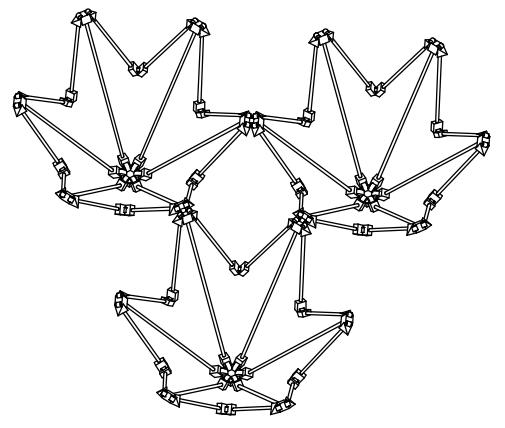

(b) General configuration

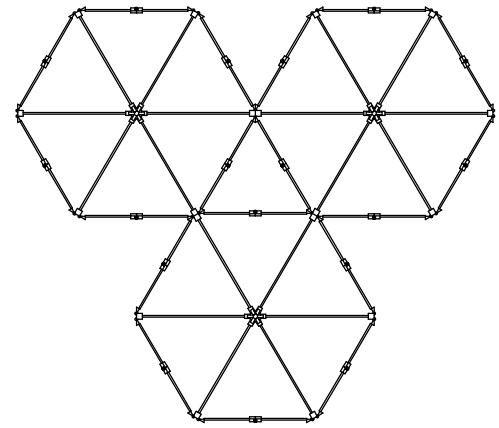

(c) Deployed configuration

Fig. 3 Large single DOF deployable network constructed from Myard deployable mechanisms

Due to the overconstrained mobility characteristic of the Myard mechanism, the mechanism is critical at the dimensional accuracy of the mechanical parts, even a small error will make the mechanism can not be functioned normally, the joint axis $\mathbf{s}_{5}$ of the revolute joint $\mathrm{E}$ should be strictly pass through the point Q such that the mechanism has a single continuous DOF, where Q locates in the symmetric plane of the Myard mechanism and the intersection line of the two planes $\left\{\mathbf{s}_{1}, \mathbf{s}_{2}\right\}$ and $\left\{\mathbf{s}_{3}, \mathbf{s}_{4}\right\}$. This is difficult to realize in the practical mechanical manufacturing, any dimensional error will make the mechanism to be a rigid structure that is not movable. As is shown in Fig. 4(a), we make the length of link $c$ to be adjustable, and it is a little longer than the theological one. Then the mechanism can not be folded onto the completely folded configuration, it becomes the rigid structure that is not movable, Fig. 4(b), therefore, the joint clearance is necessary in this kind of mechanism design.

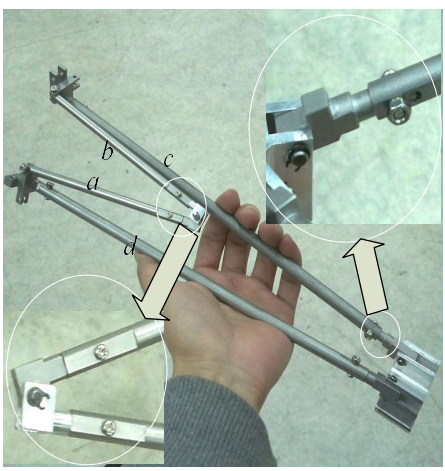

(a) Not symmetric

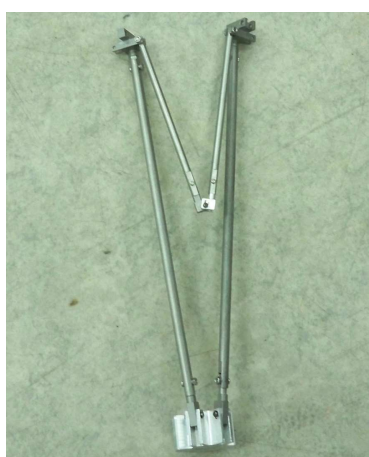

(b) Rigid structure

Fig. 4 Myard mechanism with dimensional errors 


\section{Normalized constrained model of the Myard deployable mechanism}

For the Myard 5R mechanism as shown in Fig. 1, we have to make sure that the plane $\left\{\mathbf{s}_{5}, \mathbf{O Q}\right\}$ passing through the point $\mathrm{P}$, and the point $\mathrm{Q}$ locates at the plane $\left\{\mathbf{s}_{3}, \mathbf{s}_{4}\right\}$ to ensure the single continuous mobility of the mechanism. This is difficult in the manufacturing process of the mechanical parts of this mechanism. Based on reference by Li B. et al., (2015), we can attach two revolute joints $\mathrm{F}$ and $\mathrm{G}$ along the two short links $a$ and $b$ of the original Myard 5R mechanism without changing the single mobility of the resultant mechanism, as shown in Fig. 5. In this case, joint axes of $\mathrm{F}$ and $\mathrm{G}$ intersect at a point $\mathrm{E}$ such that any rotation in the plane $\left\{\mathbf{s}_{6}, \mathbf{s}_{7}\right\}$ and passing through the point $\mathrm{E}$ can be realized, because $\mathbf{s}_{5} \cap\left\{\mathbf{s}_{6}, \mathbf{s}_{7}\right\}=\mathrm{E}$, then any rotation in the plane $\left\{\mathbf{E P}, \mathbf{s}_{5}\right\}$ and passing through the point $\mathrm{E}$ can be realized, and it is easy to find an allowable rotational axis in the plane $\left\{\mathbf{E P}, \mathbf{s}_{5}\right\}$ and passing through point $\mathrm{E}$ and also passing through the intersection line of the two planes $\left\{\mathbf{s}_{1}, \mathbf{s}_{2}\right\}$ and $\left\{\mathbf{s}_{3}, \mathbf{s}_{4}\right\}$ so that the mechanism has a single continuous DOF.

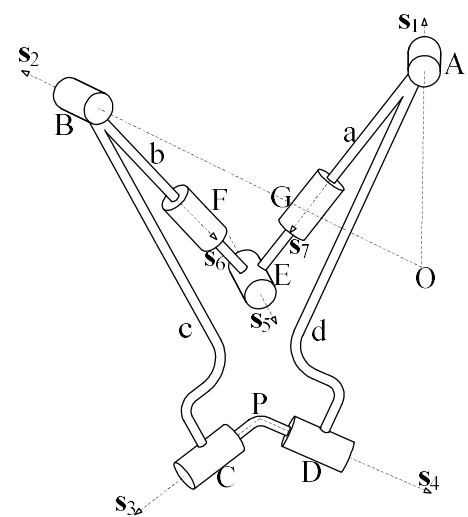

Fig. 5 Normalized-constrained mechanism of Myard 5R mechanism

Based on the general Grübler-Kutzbach mobility criterion (Grigore Gogu, 2005)

$$
F=6(n-g-1)+\sum_{i=1}^{g} f_{i}
$$

Where $n$ is the number of moving parts (or links) and one of them is the fixed base, $g$ is the number of joints, $f_{i}$ is the number of DOFs for the $i$ th joint. The DOF for the $7 \mathrm{R}$ mechanism can be calculated as

$$
F=6(7-7-1)+(7 \times 1)=1
$$

Equation (3) shows that the 7R mechanism is normal constrained. For the normalized-constrained 7R mechanism as given in Fig. 5, it will not so sensitive to the dimensional errors of the mechanical parts. We will demonstrate this through the computer-aided model (CAD) as shown in Fig. 6. Based on Eq. (1), the two short links $a$ and $b$ of the original Myard mechanism should be identical and equal to $d \sin \alpha_{52}$, otherwise, the mechanism could not be movable. However, for the normalized-constrained 7R mechanism, it does not have this requirement, as shown in Fig. 6(a) and Fig. 6(b). Besides, any possible dimensional errors in the long links $c$ and $d$, and errors in the angle of $\alpha_{23}$ and $\alpha_{34}$, are also allowable in this mechanism without changing its movable ability, as shown in Fig. 6(c) Fig. 6(f). Although this errors do not change the movable ability of the mechanism, the deployable ability of the mechanism has been changed such that the mechanism could not be deployed/folded completely, in the next section, we will use the joint clearance to solve this problem.

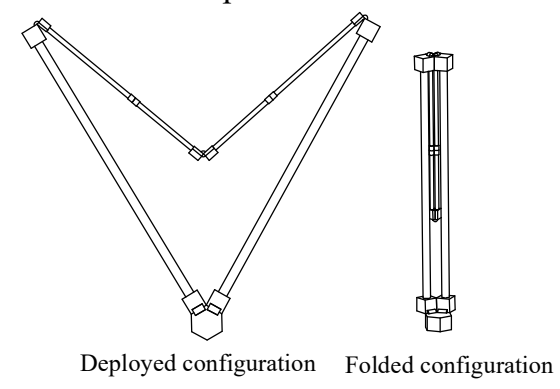

(a) Link lengths of $a$ and $b$ are greater than $d \sin \alpha_{52}$

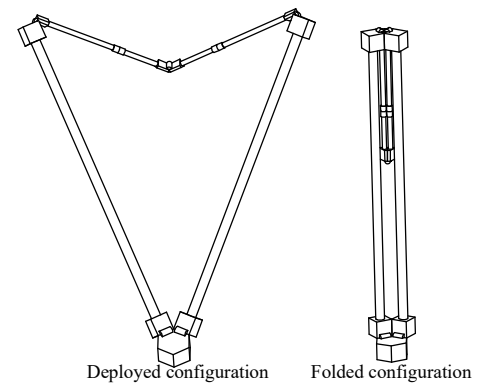

(b) Link lengths of $a$ and $b$ are smaller than $d \sin \alpha_{52}$ 


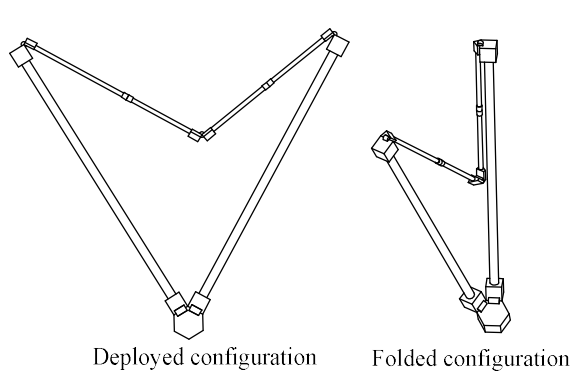

(c) Link length of $b$ is greater than $a$

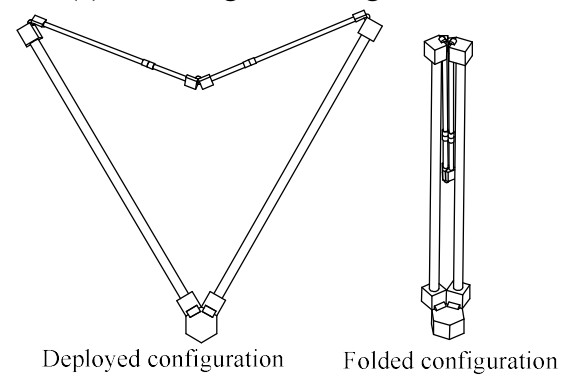

(e) $\alpha_{23} \neq \pi / 2$

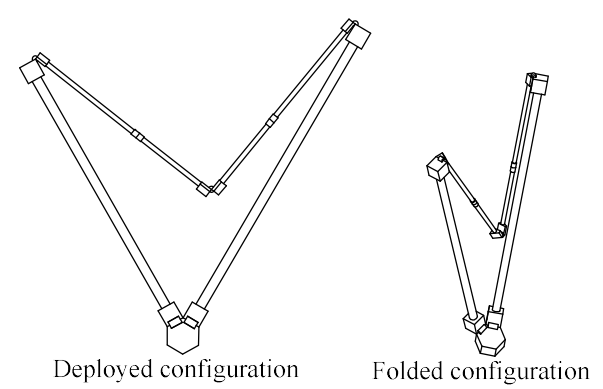

(d) Link length of $d$ is greater than $c$

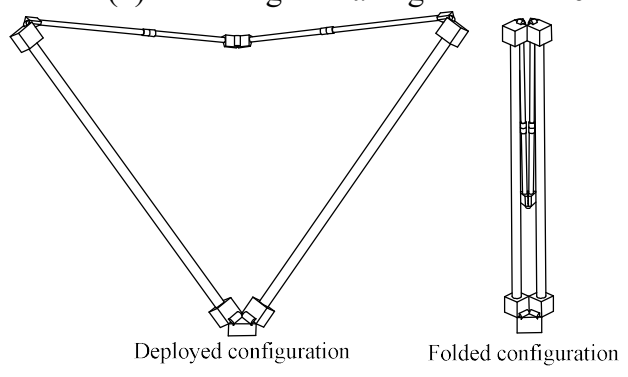

(f) $\alpha_{34} \neq 2 \pi / 3$

Fig. 6 CAD model of the normalized-constrained Myard mechanism with some dimensional errors

\section{Optimized joint clearance design for Myard mechanism}

The joint clearance can be used to release the overconstraints in the mechanism, however, the joint clearance will also bring significant influence on the performance of the deployable mechanisms. Due to the existence of the joint clearance, the counter-force appears in the kinematic pairs, and larger size of joint clearance will lead to larger contact force. The substantially vibration under contact collision will decrease the dynamic performance of the mechanism dramatically. All these will make the possibility of the unforeseen failure for the mechanical systems. Therefore, we need to design the joint with clearance but should not be too large, optimized joint clearance should be calculated.

The clearance model for the revolute joint is as shown in Fig. 7(a), a revolute joint consists of a socket and a pin in the hole of the socket. It is assume that the maximum relative translation of link $a$ and link $b$ along $\mathrm{z}$ direction due to the joint clearance direction is $2 \delta$, and the maximum relative translation of link $a$ relative to link $b$ along y direction due to the joint clearance is $2 \sigma$, then clearance of the revolute joint can be represented by the two parameters $\delta$ and $\sigma$. Based on this model, the maximum allowable rotation $\varphi$ of link $a$ relative to link $b$ around any direction perpendicular to $\mathrm{z}$ axis is shown in Fig. 7(b), which can be calculated as follow:

$$
\varphi=\angle \mathrm{ADC}-\angle \mathrm{ADB}=\arctan \left(\frac{R}{H}\right)-\arcsin \left(\frac{R-2 \sigma}{\sqrt{R^{2}+H^{2}}}\right)
$$

Where $R$ is the diameter of the socket hole, and $H$ is the height of the socket hole. Eq. (4) is calculated based on the fact that $\delta$ is big enough for its rotational motion along any direction perpendicular to $\mathrm{z}$ axis, i.e., the maximum relative translation $2 \delta$ of link $a$ and link $b$ along $\mathrm{z}$ axis should satisfy the following relationship

$$
\delta \geq(R-2 \sigma) \tan \varphi
$$

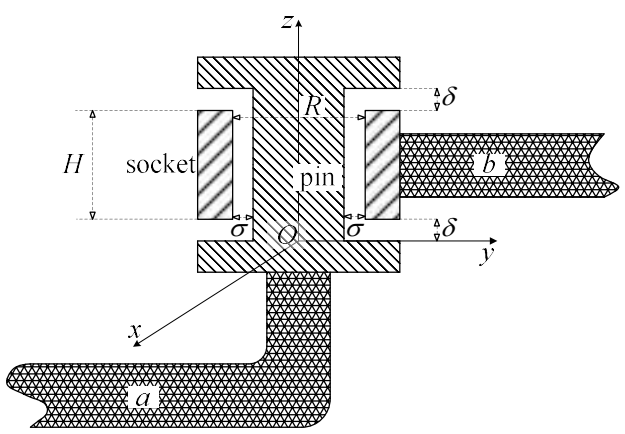

(a) Default configuration

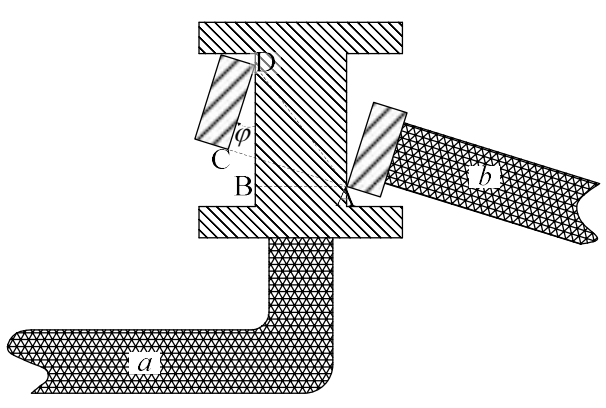

(b) Maximum allowable rotation

Fig. 7 Clearance model for a revolute joint 
Based on the normalized-constrained model of the Myard mechanism given in Fig. 5, we need to calculate the rotational range of the two joints $F$ and $G$ to design the proper joint clearance in the joint $E$ instead of using the two additional revolute joints. To realize this, we first need to find the closure equation of the normalized-constrained $7 \mathrm{R}$ mechanism as given in Fig. 5. By using the Denavit- Hartenberg (D-H) approach (Denavit J and Hartenberg R S, 1995), the D-H model of this 7R mechanism is shown in Fig. 8, axes $z_{1}, z_{2}, z_{3}, \cdots, z_{7}$ are the joint axes of C, B F, E, G, A and D (see Fig. 5) respectively, each $x_{i}$ is the common perpendicular of $z_{i}$ and $z_{i+1}$ and pointing from $z_{i}$ to $z_{i+1}$, then the D-H parameters of this mechanism is as shown in Table 1.

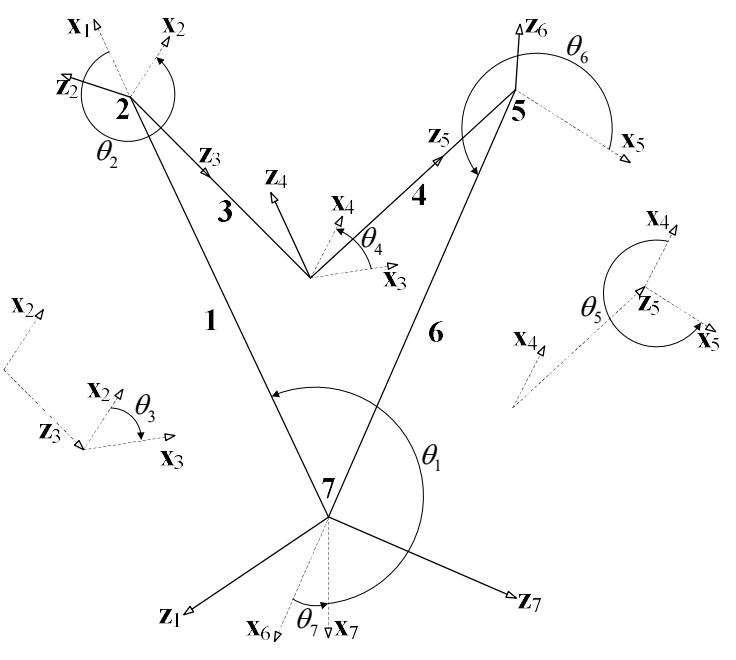

Fig. 8 Joint variables of normalized constrained Myard mechanism

Table 1 D-H parameters for the normalized constrained Myard mechanism

\begin{tabular}{c|c|c|c|c}
\hline Link number $i$ & Offset $S_{i}$ & Link length $a_{i-1}$ & Twist angle $\alpha_{i-1, i}$ & Joint variable $\theta_{i}$ \\
\hline 1 & 0 & $L$ & $-\pi / 2$ & $\theta_{2}$ \\
\hline 2 & $S$ & 0 & $-\pi / 2$ & $\theta_{3}$ \\
\hline 3 & 0 & 0 & $\pi / 2$ & $\theta_{4}$ \\
\hline 4 & $S \pm \Delta y$ & 0 & $\pi / 2$ & $\theta_{5}$ \\
\hline 5 & 0 & 0 & $-\pi / 2$ & $\theta_{6}$ \\
\hline 6 & 0 & $L \pm \Delta x$ & $\pi / 2$ & $\theta_{7}$ \\
\hline 7 & 0 & 0 & $2 \pi / 3$ & $\theta_{1}$ \\
\hline
\end{tabular}

Based on the transformation matrix

$$
{ }^{i-1} \mathbf{T}_{i}=\left[\begin{array}{cccc}
c \theta_{i} & -s \theta_{i} & 0 & a_{i-1} \\
s \theta_{i} c \alpha_{i-1, i} & c \theta_{i} c \alpha_{i-1, i} & -s \alpha_{i-1, i} & -S_{i} s \alpha_{i-1, i} \\
s \theta_{i} s \alpha_{i-1, i} & c \theta_{i} s \alpha_{i-1, i} & c \alpha_{i-1, i} & S_{i} c \alpha_{i-1, i} \\
0 & 0 & 0 & 1
\end{array}\right]
$$

we can get the closure equation of the $7 \mathrm{R}$ mechanism as

$$
\mathbf{I}={ }^{1} \mathbf{T}_{2}{ }^{2} \mathbf{T}_{3}{ }^{3} \mathbf{T}_{4}{ }^{4} \mathbf{T}_{5}{ }^{5} \mathbf{T}_{6}{ }^{6} \mathbf{T}_{7}{ }^{7} \mathbf{T}_{1}
$$

Where $\mathbf{I}$ is the $4 \times 4$ identity matrix and ${ }^{1} \mathbf{T}_{2},{ }^{2} \mathbf{T}_{3},{ }^{3} \mathbf{T}_{4},{ }^{4} \mathbf{T}_{5},{ }^{5} \mathbf{T}_{6},{ }^{6} \mathbf{T}_{7}$ and ${ }^{7} \mathbf{T}_{1}$ can be calculated by using the Eq.(6)and the values in Table 1. For example, ${ }^{1} \mathbf{T}_{2}$ is calculated as

$$
{ }^{1} \mathbf{T}_{2}=\left[\begin{array}{cccc}
\cos \theta_{2} & -\sin \theta_{2} & 0 & L \\
0 & 0 & 1 & 0 \\
-\sin \theta_{2} & -\cos \theta_{2} & 0 & 0 \\
0 & 0 & 0 & 1
\end{array}\right]
$$

Based on the model of the revolute joint as given in Fig. 7 and the closure equation given in Eq. (7), the joint clearance design procedures of the overconstrained Myard mechanism can be summarized as follows:

Step 1: Specify the lengths for the long links $c$ and $d$ (in Fig. 5) as design parameters $L$ and $L \pm \Delta x$ (in Table 1) respectively. Specify the lengths for the long links $a$ and $b$ (in Fig. 5) as design parameters $S$ and $S \pm \Delta y$ (in Table 1) 
respectively. $\Delta x$ and $\Delta y$ are the possible dimensional errors for the links.

Step 2: Specify the deployed configuration and the folded configuration, in this paper, it is assume that in the deployed configuration, all nonzero links are coplanar, in the folded configuration, part the nonzero links are collinear, the mechanism could not be completely folded due to the dimensional errors.

Step 3: Calculate the motion range of $\theta_{3}$ and $\theta_{5}$ between deployed configuration and folded configuration Based on the closure equation given in Eq. (7).

Step 4: Calculate the joint clearance based on the clearance model of revolute joint given in Fig. 7 and the Eq. (4) and Eq. (5).

\section{Design example}

In this section, we will provide a design example to illustrate the clearance design of deployable Myard mechanism. Firstly, it is assume that we want to design a Myard deployable mechanism with the two long links $c$ and $d$ equal to $300 \mathrm{~mm}$, the possible error in manufacturing of the two long links is $\pm 2 \mathrm{~mm}$. Based on Eq. (1), the theoretical length for the two short links $a$ and $b$ is $d \sin 30^{\circ}$, i.e., $150 \mathrm{~mm}$, in this design example, we will design the lengths of the two short links to be $160 \mathrm{~mm}$, which is not equals to $d \sin 30^{\circ}$, and the possible error in manufacturing of the two short links is $\pm 1 \mathrm{~mm}$. Then the design procedure can be given as follows:

(1) Based on the worst case, let lengths of $c$ and $d$ be $300-2 \mathrm{~mm}$ and 300+2mm respectively, let lengths of $a$ and $b$ be $160-1 \mathrm{~mm}$ and $160+1 \mathrm{~mm}$ respectively. Based on Eq. (1), this set of parameters do not safety a Myard mechanism, we need to use the normalized model of the Myard mechanism as given in Fig. 5 to assure the mobility of the mechanism.

(2) Suppose that in the deployed configuration, links $a, b, c$, and $d$ are coplanar, in the folded configuration, links $a$ and $d$ are collinear. The CAD model of the mechanism under the parameters given in step (1) is shown in Fig. 9 , in this two configuration, we can calculate joint variables of $\theta_{1}, \theta_{2}, \theta_{4}, \theta_{6}$ and $\theta_{7}$ by solving the quadrangle. We have $\theta_{1}=270^{\circ}, \theta_{2} \approx 49.56^{\circ}, \theta_{4} \approx 139.29^{\circ} \theta_{6} \approx 308.85^{\circ} \theta_{7}=90^{\circ}$ in deployed configuration, we have $\theta_{1}=187.11^{\circ}, \theta_{2} \approx 79.26^{\circ}, \theta_{4} \approx 22.85^{\circ}, \theta_{6}=270^{\circ}, \theta_{7} \approx 14.02^{\circ}$ in folded configuration.

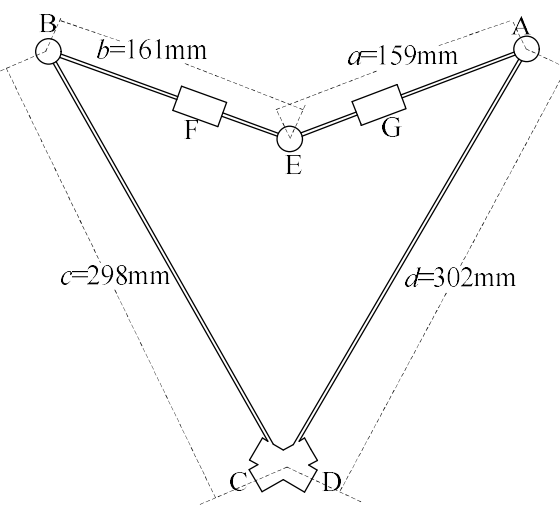

a) Deployed configuration

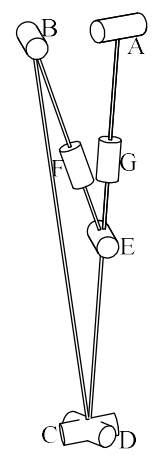

(b) Folded configuration

Fig. 9 Deployed and folded configuration of the normalized constrained Myard mechanism

(3) Based on the closure equation in Eq. (8), we can calculate the differences for the two joint variables $\theta_{3}$ and $\theta_{5}$ of joint $\mathrm{F}$ and $\mathrm{G}$ between deployed configuration and folded configuration two be $\Delta \theta_{3}=\left|\theta_{3}^{D}-\theta_{3}^{F}\right|=0^{\circ}$, $\Delta \theta_{5}=\left|\theta_{5}^{D}-\theta_{5}^{F}\right|=59.24^{\circ}$.

(4) Suppose that the parameters for the revolute joint is: $R=4 \mathrm{~mm}, H=6 \mathrm{~mm}$. Let $\varphi=59.24^{\circ}$, based on Eq. (4), we can calculate $\sigma=1.0679 \mathrm{~mm}$. Based on Eq. (5) we have $\delta \geq 0.6305 \mathrm{~mm}$. By designing the joint clearance in the joint E with $\sigma=1.0679 \mathrm{~mm}$ and $\delta=0.6305 \mathrm{~mm}$, we can eliminate the two revolute joint $\mathrm{F}$ and $\mathrm{G}$ in the normalized model of the Myard mechanism. 
We can see that the difference of $\|\mathrm{BC}-\mathrm{BE}|-| \mathrm{AD}-\mathrm{AE}\|=|| 298-161|-| 302-159 \|=6 \mathrm{~mm}$, which is much larger than $\sigma=1.0679 \mathrm{~mm}$. Then the clearance calculated in step (4) could not remedy for this error. In order to make the mechanism can be completely folded into a configuration with links $a, b, c$, and $d$ being collinear, we need re-calculate the clearance for all the joints in A, B, C D and E, if we use even distribution of the $6 \mathrm{~mm}$ error to the five joints, then we have $\sigma=1.2 \mathrm{~mm}$, and $\delta \geq 0.5465 \mathrm{~mm}$.

\section{Conclusion}

This paper has proposed a normalized-constrained approach for joint clearance design the deployable overconstrained Myard 5R mechanism, we have first shown that the joint clearance is necessary and inevitable in the design of this type of mechanisms, the normalized-constrained model of Myard mechanism has been proposed for the design of the clearance joints, which has shown that the normalized-constrained mechanism is not so sensitive to the dimensional errors of the links, possible errors will not change the movable ability of the mechanism. Based on the clearance joint model and the normalized constrained model of the Myard mechanism, we can calculate optimized clearance for the joints of deployable Myard mechanism. A design example has been used to show the detailed design procedures of the methodology developed in this paper.

\section{Acknowledgments}

This work was financially supported in part by National Science and Technology Support Program (project No. 2012BAH85F01) and in part by National Natural Science Foundation of China (project No. 51505097).

\section{References}

Denavit J and Hartenberg R S, A Kinematic Notaytion for Lower Pair Mechanisms Based on Matrices, ASME Journal of Applied Mechanics, Vol. 22, (1995), pp. 215-221.

Foster C. L. and Tinker M. L., Nurre G S. Solar-array Induced Disturbance of Hubble Space Telescope Pointing Control System. Journal of Spacecraft and Rockets, 32(4), (1995), pp.634-644.

Grigore Gogu, Mobility of mechanisms: a critical review, Mechanism and Machine Theory, vol. 40, (2005), pp.1068-1097.

Hailin Huang, Zongquan Deng, Xiaozhi Qi and Bing Li. Virtual Chain Approach for Mobility Analysis of Multiloop Deployable Mechanisms. Transactions of the ASME: Journal of Mechanical Design. 135(11), 111002, (2013).

Hailin Huang, Bing Li, Rongqiang Liu and Zongquan Deng, Randomized multi-objective optimal design of a novel deployable truss, Proceedings of the Institution of Mechanical Engineers, Part G: Journal of Aerospace Engineering, Vol. 227, No. 11, (2013).

Jian Meng, Dongjun Zhang, Zexiang Li, Accuracy Analysis of Parallel Manipulators With Joint Clearance, Journal of Mechanical Design, Vol. 131, (2009).

Li Bing, Huang Hailin, and Deng Zongquan, Mobility Analysis of Symmetric Deployable Mechanisms Involved in a Coplanar 2-Twist Screw System, ASME Journal of Mechanisms and Robotics, (2015).

L. Puiga, A. Barton and N. Rando, Review: A review on large deployable structures for astrophysics missions, Acta Astronautica, Vol. 67, Issues 1-2, (2010), pp. 12-26.

Myard F. E., Contribution à la Géométrie des Systèmes Articulés. Bulletin de la Société Mathématique de France, issue 59, (1931), pp.183-210.

Nurre G. S., Sharkey J. P. and Nelson J. D., Preserving Mission on Orbit Modification of Hubble Space Telescope Pointing Control System. Journal of Guidance, Control, and Dynamics, 18(2), (1995), pp.223-230. 